\section{Forum \\ Malaria Parasite Metabolic Pathways (MPMP) Upgraded with Targeted Chemical Compounds}

\author{
Hagai Ginsburg ${ }^{1, *}$ and \\ Alyaa M. Abdel-Haleem²,3
}

Malaria Parasite Metabolic Pathways (MPMP) is the website for the functional genomics of intraerythrocytic Plasmodium falciparum. All the published information about targeted chemical compounds has now been added. Users can find the drug target and publication details linked to a drug database for further information about the medicinal properties of each compound.

The quest for artificial antimalarial drugs was started by Paul Ehrlich in the late 19th century and has continued since then at different paces relying mostly on trial-anderror tests. This effort is necessary for counteracting the evolution of parasite resistance against drugs in use. Using animal models and then using cultures of Plasmodium falciparum, only a handful of new drugs have been developed during the past six decades, most of them with an unknown target or detailed mode of action. With the completion of the genome project of this parasite [1], it was stated that 'The genome sequence provides the foundation for future studies of this organism, and is being exploited in the search for new drugs and vaccines to fight malaria'. Since then only one gene product has been successfully used for drug development to reach clinical trials [2].

The MPMP functional genomics database (MPMP.huji.ac.il) was initiated 15 years ago and is being constantly expanded and updated based on literature publications [3]. It consists of maps and tables that organize the annotated genes of the parasite genome in a functional context. It already contains expression data and all the information about the subcellular location of annotated encoded genes. It was recently thought useful to add drugrelated data to this information resource. The procedure developed for achieving this goal is described in Figure 1. First the literature (starting in the year 2000) has been scanned using PubMed with the search string 'falciparum AND (target OR drug OR antimalarial OR antiplasmodial)'. As of October 1, 2015, more than 3300 publications (and growing at 2-5 papers/week) were retrieved. These were read and selected according to the following criteria to be admitted to the final drug list: (i) Drugs that act on an identified target such as a protein encoded by the parasite genome. (ii) Compounds that act on a process that is described in MPMP such as hemozoin or $\beta$-hematin formation, or glutathione metabolism (based on an observed reduction of glutathione level with no specified target enzyme). (iii) Drugs constructed according to protein structure-based design, be it X-ray crystallography or Protein Data Bank (PDB) analysis of target sites and ligands, were included even if they were obtained by virtual screening. (iv) Purified enzymes whose biochemical properties differed significantly from those of the host homolog were included as 'suggested target'. (v) Peptides derived from invasion-related proteins that demonstrably inhibit the invasion of erythrocytes by the parasite. (vi) Drugs and targets retrieved by system analyses or based on the topology of metabolic network. Altogether, this screening yielded 1707 relevant articles and 2317 different compounds. In several cases only the scaffolds of the new compounds are presented because there were too many variants to be shown (see for example [4]). In similar cases, only the 3-14 most active compounds were included (although reduced activity could be very revealing). (vii) Drugs which demonstrably inhibit parasite proliferation but which lack a specified target were not included although some of them could be drugs in use.

Antimalarial compounds were defined as drugs in the present context because many of them were shown to have recognized selectivity and a confirmed mode of action. Nevertheless, it should be underscored that during the past decade it was learned that many of the chemical probes in use today had initially been characterized inadequately and have since been proven to be nonselective 'or associated with poor characteristics such as the presence of reactive functionality that can interfere with common assay features' [5]. Despite the claim that attempts to disseminate accurate and reliable information to the research community regarding both well and poorly characterized compounds does not seem to be having sufficient impact, it was decided to present the malaria-related information in a target-oriented format.

Each entry in the drug database includes drug name (or suggested target), ID of the gene that encodes for the target or the name of a functional process, and the PubMed ID of the publication from which the data were obtained. Each drug is linked to a drug database such as PubChem (http:// pubchem.ncbi.nlm.nih.gov/), ChemSpider (www.chemspider.com/), MolBase (www. molbase.com/en/index.html), CheMBL (www.ebi.ac.uk/chembl/), ZINC (http:// zinc.docking.org/), and some others. Understandably, the most recent compounds are not yet present in these databases. Linking provides a wealth of information about the compounds, such as 2D and 3D structures, medical subject heading (MeSH) synonyms, as well as chemical, physical, and some pharmacological properties, PubMed crosslinks, drug and medication information, therapeutic uses, and drug vendors, as well as pharmacological and biochemical information. ChemSpider is particularly useful because it searches automatically by systematic 


\section{Trends in Parasitology}

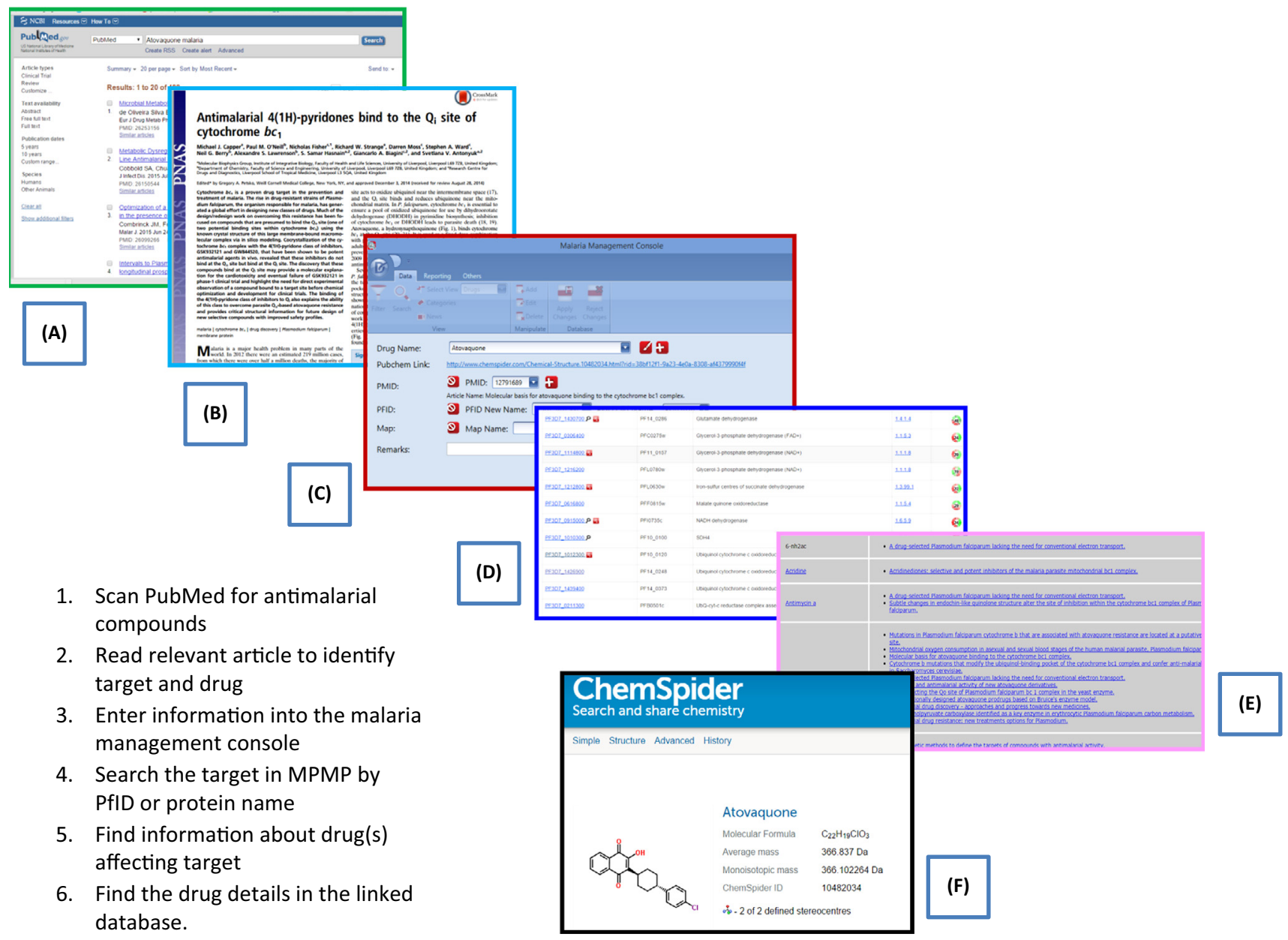

Trends in Parasitology

Figure 1. Procedure for Data Collection and Retrieval by Malaria Parasite Metabolic Pathways (MPMP). PfID, Plasmodium falciparum gene identifier.

names, synonyms, trade names, and database identifiers.

Once entered into MPMP the drugs are easily visible through another new feature that was added recently: when a map is uploaded on the screen, the full list of its constituents appears in a tabular form at the bottom. Those that serve as a drug target are indicated by an Rx icon, and pressing on the ID leads to a page that contains all the information mentioned above. The search engine can also be used to search for a drug, by drug name, and by target and metabolic pathway name. Data will be collected as they become available on the web and the site will be readily updated.

It is hoped that, as with the other elements of MPMP, antimalarial compounds with be uploaded in real time as they become available in the literature.

By compiling all antimalarial drug-related information deriving from cell-, chemistryand target-based approaches, MPMP provides a resource that assists knowledge-guided discovery and development of new drug candidates.

\section{Acknowledgments}

Software developer responsible for MPMP back-end Ofir Barlev and Web developer Alex Artyomov are wholeheartedly thanked for their relentless efforts to improve data organization and its display. The website is presently supported by the Computation Authority of The Hebrew University of Jerusalem, Israel (www.huji. ac.l//huji/eng/info_computers_e.htm), by the World Health Organization (WHO) Special Program for Research and Training in Tropical Diseases (TDR), by the Israel Academy of Sciences and Humanities, by OzEMalaR, and has received support in the past from BioMalPar and from EviMalaR.

${ }^{1}$ Department of Biological Chemistry, Institute of Life Sciences, The Hebrew University of Jerusalem, Jerusalem 91904, Israel 


\section{Trends in Parasitology}

${ }^{2}$ Computational Bioscience Research Center, King Abdullah University of Science and Technology (KAUST), Thuwal 23955-6900, Saudi Arabia

${ }^{3}$ Biological and Environmental Sciences and Engineering (BESE) Division, King Abdullah University of Science and Technology (KAUST), Thuwal 23955-6900, Saudi Arabia

${ }^{*}$ Correspondence: hagai.ginsburg@gmail.com (H. Ginsburg) http://dx.doi.org/10.1016/j.pt.2015.10.003

References

1. Gardner, M.J. et al. (2002) Genome sequence of the human malaria parasite Plasmodium falciparum. Nature 419, 498 511

2. Phillips, M.A. et al. (2015) A long-duration dihydroorotate dehydrogenase inhibitor (DSM265) for prevention and treatment of malaria. Sci. Transl. Med. 7, 296ra111
3. Ginsburg, H. (2006) Progress in in silico functional genomics: the malaria Metabolic Pathways database. Trends Parasitol. 22, 238-240

4. Liu, Y. et al. (2011) Synthesis and antimalarial activity of novel dihydro-artemisinin derivatives. Molecules 16, 4527-4538

5. Arrowsmith, C.H. et al. (2015) The promise and peril of chemical probes. Nat. Chem. Biol. 11, 536-541 